\title{
Juvenile breast hypertrophy
}

\author{
Benedita Bianchi de Aguiar ${ }^{1}$, Rita Santos Silva², Carla Costa ${ }^{2}$, Cintia Castro-Correia ${ }^{2}$, Manuel Fontoura ${ }^{2}$
}

${ }^{1}$ Paediatrics Department, Centro Hospital Entre Douro e Vouga, Santa Maria da Feira, Portugal

${ }^{2}$ Paediatric Endocrinology, Centro Hospitalar Universitário de São João, Porto, Portugal

Key words: adolescent; breast; hypertrophy

Juvenile breast hypertrophy (JBH), also called virginal hypertrophy or macromastia, is a rare benign condition, in which one or both breasts undergo a massive increase in size during puberty, usually around menarche. We present a clinical case of $\mathrm{JBH}$ and discuss the available therapeutic options.

An 11-year-old female patient was referred to our paediatric endocrinologist consultant due to breast hypertrophy. She referred with rapid growth of both breasts with a two-month evolution and no other symptoms. She presented excessive breast enlargement, with inflammatory signs of the peri-areolar skin, intense erythema, as well as bilateral tenderness. She was medicated with broad-spectrum antibiotics and was forwarded to our paediatric endocrinologist consultant. Menarche had occurred less than a week earlier. Under our observation she had massive bilateral breast hypertrophy (Fig. 1) with no palpable masses and no other alterations to her physical examination. She complained about breast pain and social embarrassment. Hormonal levels - including oestradiol, progesterone, LH, FSH, and prolactin, were normal, and tumour markers were

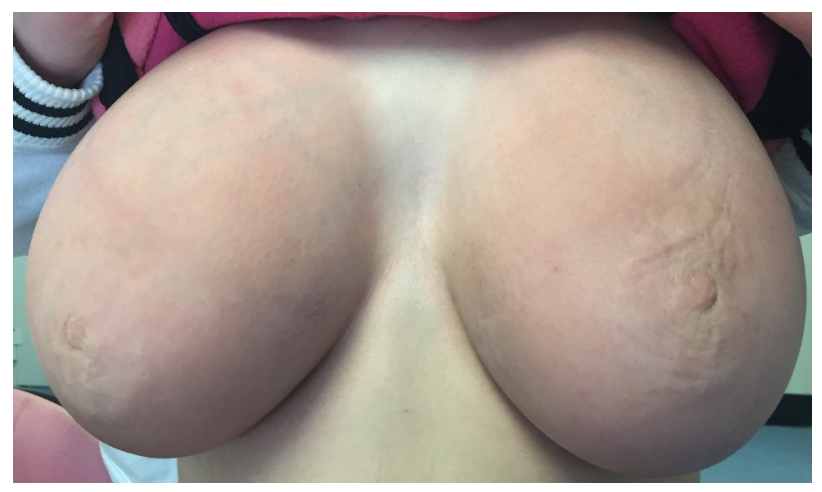

Figure 1. Juvenile breast hypertrophy (JBH) - massive bilateral breast hypertrophy in an 11-year-old patient negative. The breast ultrasound showed hypertrophy of the breast tissue bilaterally with signs of interstitial and subcutaneous oedema. Pelvic ultrasound revealed no abnormalities. She was referred to a psychology as well as a plastic surgery consultant.

Juvenile breast hypertrophy $(\mathrm{JBH})$ was first described in 1669 [1, 2]. It is characterised by rapid unilateral or bilateral disproportional overgrowth of the breasts during the peripubertal period [4]. Most cases are sporadic with rare familial incidence $[2,3]$. The classical history is a sudden increase in breast size shortly after telarche $[2,4]$. Although it is of unclear aetiology, the most popular theories include end-organ hypersensitivity to normal levels of circulating oestrogen, increased oestrogen or progesterone receptor expression, imbalance of endogenous hormone production, and excessive local oestrogen production [1, $3,4]$. Recent studies have suggested a genetic basis for this disease, involving the phosphatase and tensin homologue tumour suppressing gene, although clinical correlation is still unclear $[4,5]$. Juvenile breast hypertrophy is a benign condition, but this diagnosis can be made only after a detailed history, thorough physical examination, and after excluding underlying conditions such as endocrine and malignant disorders. Physical examination may reveal skin changes including erythema, inflammation, striae, thinning, and prominent veins [4]. In addition to the physical hazards (neck, shoulder, and back pain are frequent complaints), this condition may entail numerous psychological consequences, due to the enlarged breast mass in such a critical developmental phase, and patients frequently complain of embarrassment and social isolation [5]. Laboratory tests including endocrinology profile are not routinely indicated because they rarely reveal abnormalities [2,5]. On the other hand, breast imaging (breast ultrasound or MRI) is 
extremely important in order to exclude tumours, although in this particular condition it presents several limitations due to dense breast tissue $[2,5]$. Spontaneous resolution is rare, and the definitive treatment and its timely management is the most challenging aspect of JHB $[2,5]$. Therapeutic options include surgical management and medical therapy, although there is no formal guideline [2-4].

The medical treatment consists of hormone-modulators, which, at best, may lead to breast growth arrest without size reduction or symptom alleviation [5]. Drugs such as tamoxifen, dydrogesterone, medroxyprogesterone, bromocriptine, and danazol have been used in different cases, with limited results and questionable long-term safety. Tamoxifen is a selective oestrogen receptor modulator and the most commonly used [2-5] as well as the most effective [4, 5]; however, there is no evidence-based recommendation concerning the dosage and duration of therapy [7]. The most common surgical therapy consists of reduction mammoplasty, which must be delayed until full breast development because there is a high rate of recurrence when growth stability is not achieved prior to surgery, leading to subsequent surgical procedures associated with significant morbidity [3-5]. The most accepted surgical treatment consists of breast reduction followed by mastectomy with implant reconstruction in cases of recurrence [2]. However, there are several cases of success with breast-reduction surgery [1], and it was our option for this particular patient. A combination of both medical and surgical treatment has also been conducted in some patients, with variable results - in some cases breast enlargement developed after tamoxifen was stopped $[4,5]$. Because JBH results in severe psychosocial problems, it is of paramount importance for these adolescents to have psychological monitoring during this difficult period because hardships with body image, poor self-esteem, and difficulty fitting in are often present.

\section{References}

1. Akmal H, Marzida AL, Normala B. Juvenil Breast Hypertrophy: A successful breast reduction of $14.9 \%$ body weight without recurrence in a 5-year follow-up. Case Rep Surg. 2017; 2017(3491012), doi: 10.1155/2017/3491012, indexed in Pubmed: 28255494.

2. Karagüzel G, Bilen S, Karaçal N, et al. Virginal Breast Hypertrophy: Different Presentations of 2 Cases and the Role of Tamoxifen as an Adjuvant Therapy. J Pediatr Adolesc Gynecol. 2016; 29(5): e71-e74, doi: 10.1016/j. jpag.2016.03.008, indexed in Pubmed: 27079913.

3. Hoppe I, Patel P, Singer-Granick C, et al. Virginal Mammary Hypertrophy: A Meta-Analysis and Treatment Algorithm. Plast Reconstr Surg. 2011; 127(6): 2224-2231, doi: 10.1097/prs.0b013e3182131bd1, indexed in Pubmed: 21617457.

4. Wolfswinkel EM, Lemaine V, Weathers WM. Hyperplastic Breast Anomalies in the Female Adolescent Breast. Semin Plast Surg. 2013; 27(1): 49-55, doi: 10.1055/s-0033-1347167, indexed in Pubmed: 24872740.

5. Baker SB, Burkey BA, Thornton P, et al. Juvenile gigantomastia: presentation of four cases and review of the literature. Ann Plast Surg. 2001; 46(5): 517-25; discussion 525, doi: 10.1097/00000637-200105000-00011 indexed in Pubmed: 11352426. 